\title{
Tratamento Percutâneo por Cateter da Degeneração Valvar Biológica Aórtica - O valve-in-valve Aórtico
}

\author{
Percutaneous Catheter Treatment of Biological Aortic Valve \\ Degeneration - The Aortic valve-in-valve \\ Adriano Dias Dourado Oliveira ${ }^{1^{*}}$, Ricardo Peixoto Oliveira ${ }^{1}$ \\ Serviço de Cardiologia Intervencionista do Hospital Santa Izabel da Santa Casa da Bahia; \\ Salvador, Bahia, Brasil
}

Correspondence addresses: Dr. Adriano Dourado Oliveira addoliveira@hotmail.com

Received: September 18, 2021

Revised: November 27, 2021

Accepted: December 14, 2021

Published: December 28, 2021

Data Availability Statement: All relevant data are within the paper and its Supporting Information files.

Funding: This work was the result of authors' initiative. There was no support of research or publication funds.

Competing interests: The authors have declared that no competing interests exist.

\section{Copyright}

(C) 2021 by Santa Casa de Misericórdia da Bahia.

All rights reserved.

ISSN: 2526-5563

e-ISSN: 2764-2089

DOI: 10.35753
A doença valvar perfaz importante causa de morbidade cardiovascular. $O$ envelhecimento populacional associado a maior número de trocas valvares com uso de bioprótese, considerando a durabilidade destas, determinou um aumento de pacientes candidatos a reintervenção por degeneração valvar. Esses pacientes, recentemente, passaram a ter o implante percutâneo de válvula aórtica como alternativa à cirurgia em condições de alto risco cirúrgico. Descreve-se, neste cenário, o papel do implante valvar transcateter em pacientes com prótese biológica degenerada, considerando aspectos relacionados a indicação e resultados do procedimento.

Palavras-chave: TAVR; valve-in-valve; Degeneração Valvar.

Valvular disease is a critical cause of cardiovascular mortality. The rise of the elderly population related to a high account of surgeries using bioprosthesis, considering their durability, has been associated with an increase of patients undergoing procedures to the valvular failure. Recently, these patients have become candidates for transcatheter aortic valve replacement as an alternative to conventional surgery in high-risk profiles. It is described the role of percutaneous valve implantation in patients with bioprosthesis degeneration, considering aspects related to indication and long-term follow-up.

Keywords: TAVR; valve-in-valve; Bioprosthesis Degeneration.

\section{Introdução}

A melhoria na expectativa de vida da população mundial fez surgir doenças relacionadas ao envelhecimento. Algumas dessas condições clínicas são responsáveis pelo aumento da mortalidade cardiovascular nessa população de idosos. ${ }^{1}$ Nesse cenário, destacam-se as doenças valvares cardíacas, envolvendo as válvulas nativas e/ou degeneração tardia de implantes valvares cirúrgicos ou percutâneos. ${ }^{2}$

A partir do primeiro implante percutâneo por cateter de prótese valvar aórtica (TAVR) para tratamento de estenose aórtica calcificada, ${ }^{3}$ por Dr. Alain Cribier, em 2002, houve ampla validação e expansão do método para populações de riscos cirúrgicos diversos, tendo se tornado o método de eleição em pacientes considerados inoperáveis, ${ }^{4}$ ou com alto risco ${ }^{5,6}$ e alternativa plausível em pacientes de risco intermediário ${ }^{7,8}$ e até baixo. ${ }^{9,10}$ 
Nesse novo paradigma, o grupo de pacientes portadores de disfunção de bioprótese valvar aórtica (previamente submetidos a intervenção por toracotomia), que naturalmente possuem risco cirúrgico aumentado (segunda ou terceira reoperação, disfunção ventricular, disfunção renal, idade, ou a combinação destes) passaram a ser candidatos ao tratamento percutâneo por cateter, comumente denominado valve-in-valve (ViV) aórtico ou TAVR-in-valve.

Nesta revisão, será abordado o tratamento percutâneo por cateter da disfunção de bioprótese valvar aórtica.

\section{A Magnitude do Problema}

A degeneração valvar aórtica nativa, seja por insuficiência ou estenose, é uma importante causa de insuficiência cardíaca em todo mundo. Estimase, por exemplo, que até $5 \%$ da população com idade superior a 65 anos apresenta estenose aórtica, ${ }^{2}$ perfazendo um contingente significativo quando se considera o envelhecimento populacional global.

A cirurgia cardíaca com troca da válvula nativa por prótese biológica ou mecânica é a terapêutica clássica, já bem estabelecida, havendo diferenças significativas entre as biopróteses e as próteses mecânicas. As próteses mecânicas têm como principal benefício a durabilidade e como maior revés a necessidade de utilização de anticoagulantes inibidores da vitamina K. Esses medicamentos apresentam imprevisibilidade farmacodinâmica e de interação medicamentosa, associados aos riscos de eventos hemorrágicos. Nos últimos anos, temse observado importante redução da indicação de próteses mecânicas. ${ }^{11}$ Nos EUA, por exemplo, entre 2008-2017, a indicação de prótese mecânica aórtica reduziu de $45,3 \%$ para $17 \%$ dos casos e mitral de $59,5 \%$ para 29,2\% (Figura 1 ).

Reconhecendo-se a perda de resultado a longo prazo das biopróteses valvares cirúrgicas, entre 10-20 anos ${ }^{12}$ e a melhoria na expectativa de vida da população, era previsto um aumento significativo de pacientes necessitando de novas intervenções por disfunção valvar. A cada nova abordagem cirúrgica há incremento do risco perioperatório e a TAVR-in-valve surge como alternativa menos invasiva.

O primeiro caso TAVR-in-valve foi relatado em 2007 e desde então esse procedimento tem experimentado expressiva expansão, com adesão ainda limitada decorrente das particularidades anatômicas da sua indicação e durabilidade ainda sob avaliação. ${ }^{13}$

Figura 1. Tendência temporal de indicação de próteses biológicas ou mecânicas nos Estados Unidos, entre 2008-2017.

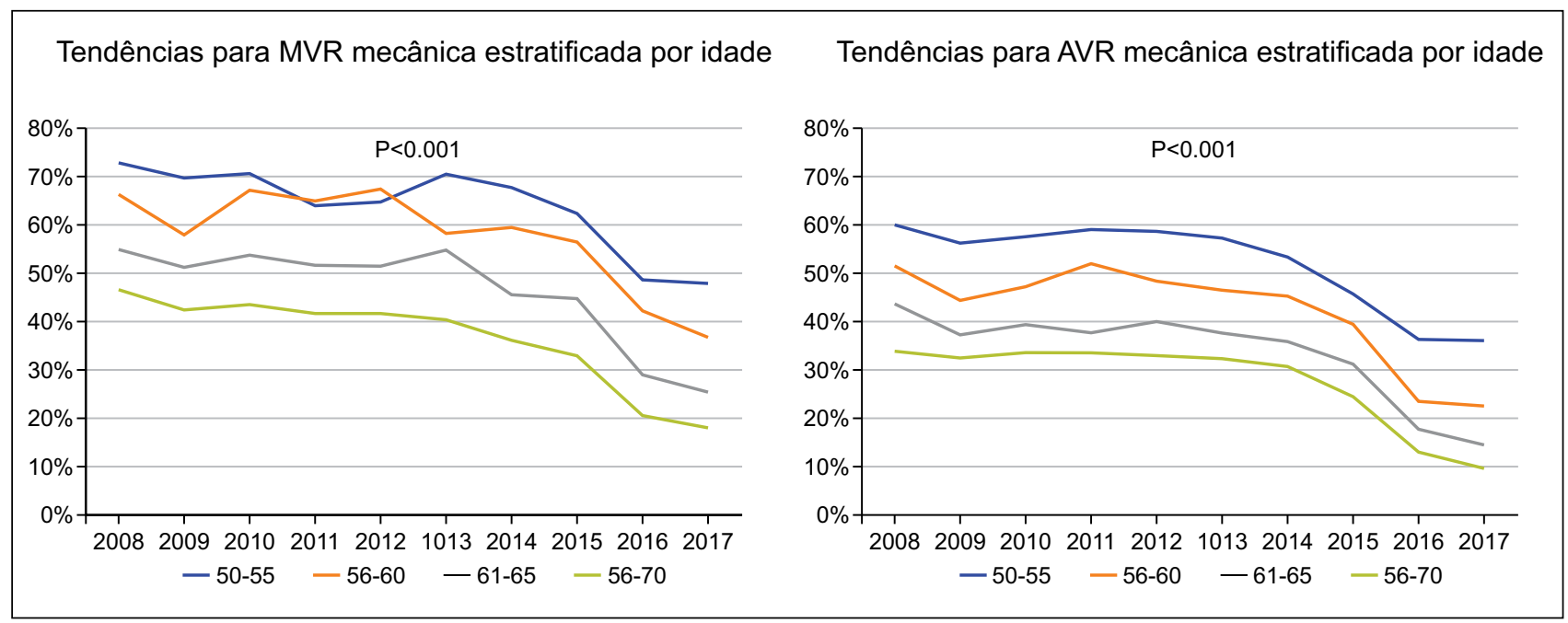

Fonte: Carroll and colleagues (2020). ${ }^{11}$ 


\section{Biopróteses Cirúrgicas: Um Fator a Ser Considerado}

As biopróteses cirúrgicas, no momento, são as próteses candidatas à avaliação para TAVR$\mathrm{ViV}$, apesar de já existirem relatos isolados de intervenções em prótese metálica, em condições extremas. ${ }^{14}$ São geralmente compostas de pericárdio bovino ou porcino, construídas sobre um aparato anelar (stented), posicionadas diretamente no anel valvar por sutura (stentless), ou sem sutura (sutureless). Os folhetos, por sua vez, podem ser suturados na face interna ou externa da prótese. Tais características tem impacto no perfil hemodinâmico da prótese o no diâmetro interno. ${ }^{15} \mathrm{~A}$ presença do anel (stent) tem impacto na factibilidade do procedimento (possibilidade ou não de fratura) e a área luminal interna da prótese tem importância na predição do comportamento hemodinâmico das próteses ViV.

Existem aplicativos, disponíveis em plataformas digitais descrevendo os tipos de prótese e suas características, que auxiliam e são amplamente utilizados no planejamento da intervenção (app ViV Aortic).

A disfunção da bioprótese, por sua vez, pode acontecer por diversos mecanismos: degeneração estrutural, alteração intrínseca e permanente dos folhetos ou estrutura de sustentação da prótese; disfunção não estrutural, como por exemplo a regurgitação paravalvar, a desproporção pacienteprótese, deiscência de sutura e redução extrínseca da mobilidade dos folhetos por formação de pannus; trombose; endocardite. A degeneração estrutural, a principal indicação para TAVRin-valve, ocorre pela resposta inflamatória ao longo dos anos, com posterior deposição de cálcio. ${ }^{16}$

\section{Indicação}

Considera-se, para efeito de indicação de implante ViV aórtico, pacientes desenvolvendo insuficiência cardíaca secundária à disfunção da bioprótese por degeneração estrutural, levando a estenose e/ou insuficiência importantes. A Sociedade Europeia de Cardiologia recomenda essa terapêutica como alternativa à cirurgia, em casos selecionados. ${ }^{2}$ A Sociedade Brasileira de Cardiologia, em sua diretriz, recomenda a intervenção valve-in-valve em pacientes com risco cirúrgico elevado ou inoperáveis. ${ }^{17}$

O planejamento do procedimento envolve, dentre muitos aspectos, a avaliação clínica do paciente. Utiliza-se de escores para estimativa de risco para cirurgia convencional, como o STS Score e o EuroSCORE, e escores de fragilidade clínica, dada correlação com mortalidade e risco de futilidade (Figura 2). Esses escores são acessíveis em ferramentas digitais disponíveis gratuitamente em páginas eletrônicas. ${ }^{18}$ Associado à avaliação clínica, é importante o conhecimento da prótese previamente implantada e suas características estruturais.

Nesse momento, ressalta-se a importância da angiotomografia ${ }^{19}$ para a programação do procedimento, pois permite aferição adequada da estrutura protética e de todas as estruturas adjacentes, do plano valvar à aorta ascendente, além do estudo das coronárias e das principais vias de acesso. É ferramenta fundamental para avaliação dos riscos de potenciais e graves complicações, como a oclusão de coronárias, e para escolha do tamanho de prótese. É mister lembrar a necessidade de realizar o exame sincronizado com o ECG, protocolo semelhante à angiotomografia de coronárias, de preferência em tomógrafos com no mínimo 128 canais. Tem como limitação a utilização de contraste iodado em pacientes com risco de disfunção renal.

\section{Segurança e Eficácia do TAVR-ViV Aórtico}

Dados dos registros e séries de casos publicados mostram o predomínio de pacientes de alto risco cirúrgico (avaliado pelo STS). As próteses mais frequentemente usadas foram as autoexpansiveis da família Evolut (Medtronic), e as balão-expansíveis, da família Sapien (Edwards). 
A mortalidade é inferior a $5 \%$ em 30 dias, ${ }^{20} \mathrm{com}$ baixas taxas de AVC e de reintervenção (até $7 \%$ em 08 anos). ${ }^{21}$ A sobrevida em 3 anos foi de $67,3 \%$ a $73 \%$. No registro multicêntrico de TAVR-in-Valve (Vivid Registry), com mais de 1.000 pacientes acompanhados em até 8 anos, os preditores independentes de mortalidade por todas as causas foram: próteses cirúrgicas menores, pacientes mais velhos, menor fração de ejeção, insuficiência renal crônica, diabete melito e acesso não femoral. ${ }^{19}$ Os preditores de eventos ${ }^{20-22}$ (Tabela 1), ressaltando-se que o emprego de próteses balão-expansíveis tem maior relação com necessidade de reintervenção.

A complicação mais desafiadora do procedimento é a oclusão coronariana, com elevado impacto da mortalidade. ${ }^{23}$ Ocorre em decorrência do deslocamento do folheto da prótese cirúrgica previamente implantada em direção ao óstio coronariano. O maior preditor para essa

Tabela 1. Preditores de complicações no ViV aórtico (achados do registro Vivid).

\begin{tabular}{ll}
\hline Preditores & Eventos Adversos \\
\hline Via de acesso não-transfemoral & Maior taxa de sangramentos e morte \\
Mismatch prótese cirúrgica & $\begin{array}{l}\text { Maior gradiente e necessidade de } \\
\text { reintervenção }\end{array}$ \\
Prótese balão-expansível & $\begin{array}{l}\text { Maior gradiente e necessidade de } \\
\text { reintervenção }\end{array}$ \\
VTC $<4 \mathrm{~mm}$ & Risco de oclusão coronariana \\
Tamanho do diâmetro interno $<20 \mathrm{~mm}$ & Maior mortalidade \\
\hline
\end{tabular}

Figura 2. Escore de fragilidade em TAVR e sua relação com mortalidade periprocedimento.

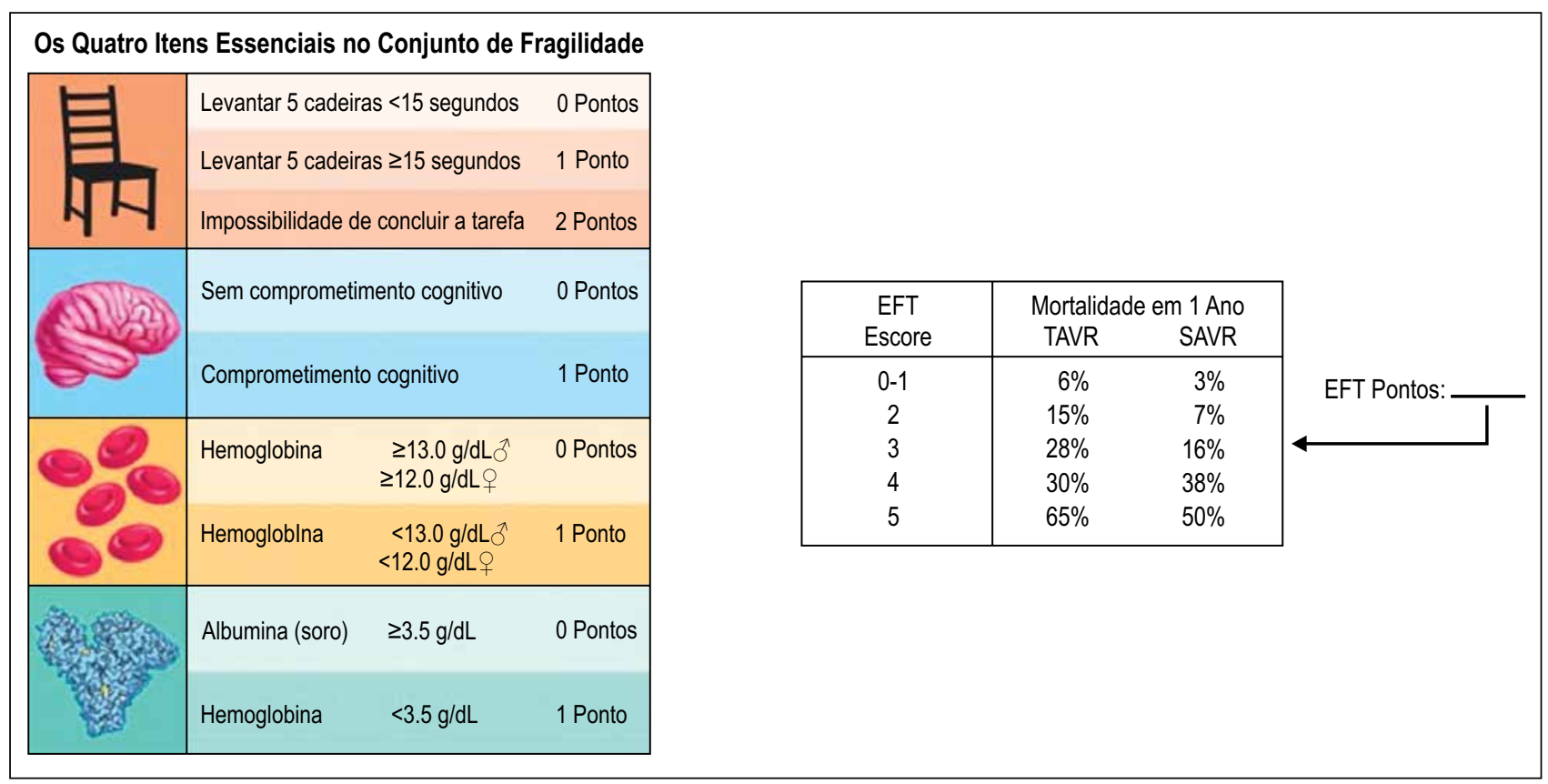

Fonte: Afilalo and colleagues. ${ }^{18}$ 
complicação é a distância virtual da posição da prótese percutânea a ser implantada para o óstio da coronária (VTC), quando inferior a $4 \mathrm{~mm} \cdot{ }^{23}$

Existem técnicas desenvolvidas em casos com alto risco de oclusão coronariana, como a laceração percutânea do folheto da bioprótese (BASILICA) ${ }^{24}$ de complexidade elevada e relacionada a maior risco de acidente vascular cerebral (AVC). Além dessa, a técnica de CHAMINÉ, em que se implanta o stent do segmento proximal da coronária, protuindo para o seio valvar e paralelo à prótese valvar percutânea, criando um canal para manutenção do fluxo coronariano (Figura 3).

Outro elemento importante a ser considerado se refere à ocorrência de gradiente transvalvar residual elevado, sugestivo de desproporção paciente-prótese. Esse achado se relaciona a pior prognóstico no seguimento, necessidade de nova intervenção e redução na sobrevida dos pacientes. ${ }^{25}$ A incidência é maior em pacientes com mismatch prévio. Esse problema pode ser contornado com uso de próteses supra-anulares, ou com implantes mais altos. Outra técnica utilizada é a fratura com

Figura 3. Técnica de Chaminé para proteção de óstio de coronárias.

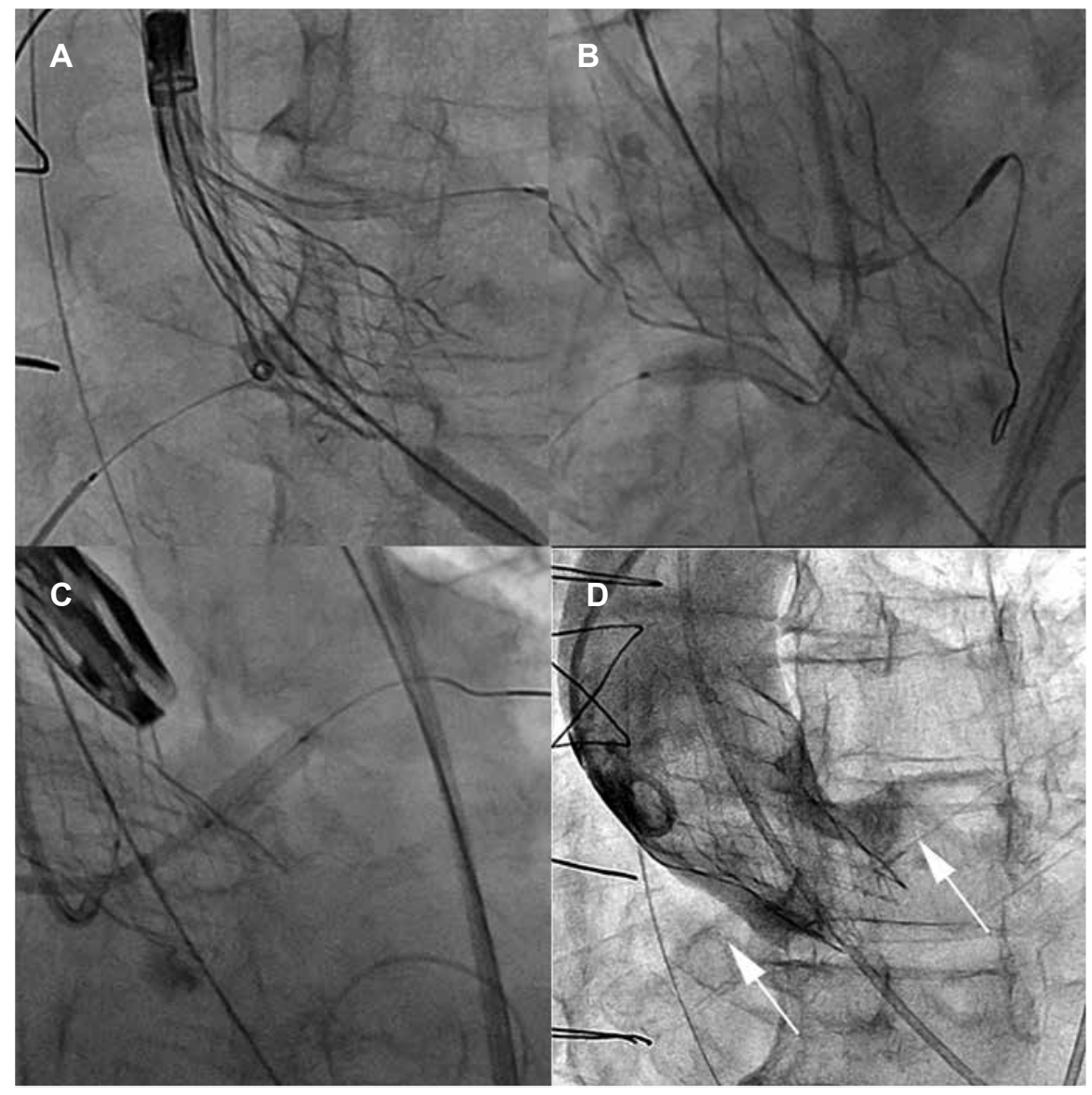

Fonte: Acervo pessoal. Trata-se de paciente de 83 anos, com passado de troca valvar aórtica em 2005, evoluindo em 2021 com insuficiência cardíaca por dupla disfunção de prótese. Fração de ejeção preservada. A. prótese sendo liberada em posição supra-anular com proteção de coronárias (VTC $<4 \mathrm{~mm}$ para ambas coronárias. B/C. Liberação de stent em óstio de coronária direita e tronco de coronária esquerda (técnica de chaminé), liberando fluxo para coronárias. D. Angiografia final demonstrando perfusão coronariana adequada. 
consequente aumento do anel da prótese biológica cirúrgica. Para tanto é necessário uso de balões de alta pressão, porém, nem todas as próteses cirúrgicas permitem a fratura. Pensando nisso, as próteses cirúrgicas mais recentes já estão sendo fabricadas com mecanismo que permite aumento de seu diâmetro, sem necessidade de fratura.

A ocorrência de trombose da prótese percutânea parece ser mais prevalente que da prótese cirúrgica, tendo alguns autores sugerindo anticoagulação por 3-6 meses após o procedimento. ${ }^{13}$

\section{Resultados Comparativos entre TAVR-in-valve e Cirurgia}

Não existem ensaios clínicos randomizados comparando a TAVR-in-valve com uma nova intervenção cirúrgica. Todos os dados e resultados comparativos baseiam-se em estudos observacionais, fato que merece atenção, diante do provável viés de seleção de populações de riscos diferentes. De modo geral, os pacientes submetidos a nova intervenção cirúrgica são mais jovens e de menor risco. No entanto, avaliações baseadas em pareamento por escores de propensão sugerem que os desfechos duros são similares entre os dois grupos, no seguimento a longo prazo. Observa-se uma maior incidência de complicações e mortalidade precoce com o grupo cirúrgico e maior necessidade de reinternação por insuficiência cardíaca no grupo transcateter (Figura 4). ${ }^{26,27}$

\section{Conclusões}

Em pacientes portadores de disfunção de prótese aórtica, o implante percutâneo de prótese valve-in-valve aórtico posiciona-se como alternativa exequível e eficaz, com consolidada experiência em pacientes de alto risco cirúrgico. A decisão deve envolver a compreensão do Heart Team, após adequada avaliação clínica e planejamento anatômico. Os resultados a longo prazo, no que tange à durabilidade da prótese e necessidade de uma terceira intervenção, ainda são pouco conhecidos. Aguardam-se estudos randomizados para avaliação da intervenção percutânea versus nova cirurgia em pacientes de menor risco.

Figura 4. Desfechos comparando ViV e reoperação em metanálise incluindo 16 mil pacientes.

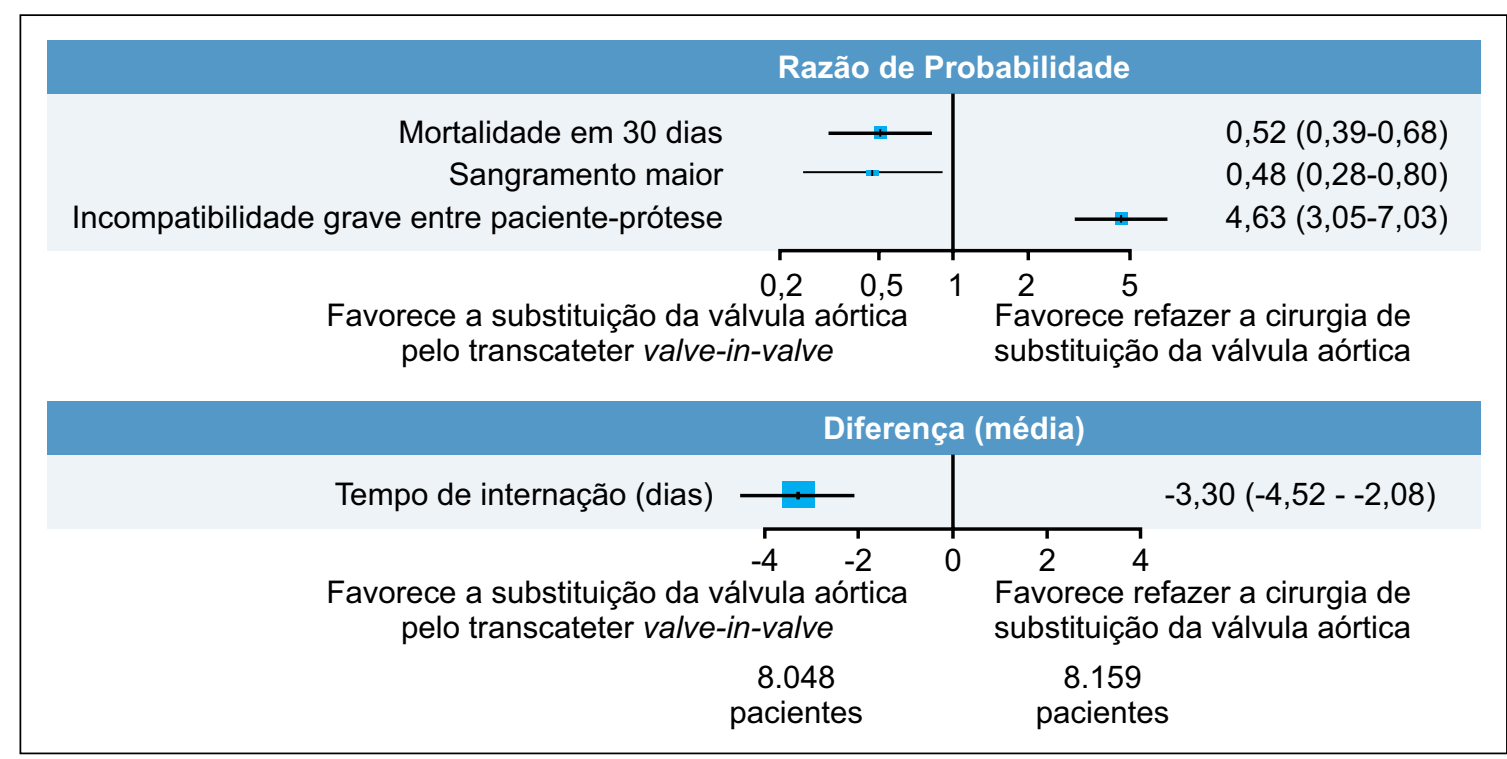

Fonte: Sá and colleagues. ${ }^{27}$ 


\section{Referências}

1. Amini M, Zayeri F, Salehi M. Trend analysis of cardiovascular disease mortality, incidence, and mortality-to-incidence ratio: results from global burden of disease study 2017. BMC Public Health 2021;21(1):1-12.

2. Vahanian A, Beyersdorf F, Praz F, Milojevic M, Baldus S, et al. $2021 \mathrm{ESC} / \mathrm{EACTS}$ Guidelines for the management of valvular heart disease. Eur Heart J 2021;1-72.

3. Cribier A, Eltchaninoff $\mathrm{H}$, Bash A, Borenstein N, Tron C, Bauer F, et al. Percutaneous transcatheter implantation of an aortic valve prosthesis for calcific aortic stenosis: First human case description. Circulation 2002;106(24):3006-8.

4. Craig R. Smith, Martin B. Leon, Michael J. Mack, D. Craig Miller, et al. Transcatheter aortic-valve implantation for aortic stenosis in patients who cannot undergo surgery. N Engl J Med 2011;365:883-91.

5. Craig R. Smith, Martin B. Leon, Michael J. Mack, et al. Transcatheter versus surgical aortic-valve replacement in high-risk patients. N Engl J Med 2011;365:883-91.

6. Adams DH, Popma JJ, Reardon MJ, Yakubov SJ, Coselli JS, Deeb GM, et al. Transcatheter aortic-valve replacement with a self-expanding prosthesis. $\mathrm{N}$ Engl J Med [Internet] 2014;370(19):1790-8. Available from: http://www.nejm.org/doi/10.1056/NEJMoa1400590

7. Leon MB, Smith CR, Mack MJ, Makkar RR, Svensson LG, Kodali SK, et al. Transcatheter or surgical aortic-valve replacement in intermediaterisk patients. N Engl J Med [Internet] $2016 \mathrm{Apr}$ 2;374(17):1609-20. Available from: https://doi. org/10.1056/NEJMoa1514616.

8. Reardon MJ, Van Mieghem NM, Popma JJ, Kleiman NS, Søndergaard L, Mumtaz M, et al. Surgical or transcatheter aortic-valve replacement in intermediaterisk patients. N Engl J Med [Internet] 2017 Mar 17;376(14):1321-31. Available from: https://doi. org/10.1056/NEJMoa1700456.

9. Mack MJ, Leon MB, Thourani VH, Makkar R, Kodali SK, Russo M, et al. Transcatheter aortic-valve replacement with a balloon-expandable valve in low-risk patients. N Engl J Med [Internet] 2019 Mar 16;380(18):1695-705. Available from: https://doi. org/10.1056/NEJMoa1814052.

10. Popma JJ, Deeb GM, Yakubov SJ, Mumtaz M, Gada H, O'Hair D, et al. Transcatheter aortic-valve replacement with a self-Expanding valve in low-risk patients. $\mathrm{N}$ Engl $\mathrm{J}$ Med [Internet] 2019 Mar 16;380(18):1706-15. Available from: https://doi.org/10.1056/NEJMoa1816885.

11. Carroll JD, Mack MJ, Vemulapalli S et al. STS-ACC TVT registry of transcatheter aortic valve replacement. J Am Coll Cardiol 2020;76:2492-2516.
12. Bourguignon T, El Khoury R, Candolfi P, Loardi C, Mirza A, Boulanger-Lothion J, Bouquiaux-StabloDuncan AL, Espitalier F, Marchand M, Aupart M. Very long-term outcomes of the carpentier-Edwards perimount aortic valve in patients aged 60 or younger. Ann Thorac Surg 2015 Sep;100(3):853-9. doi: 10.1016/j.athoracsur.2015.03.105. Epub 2015 Jul 14. PMID: 26187006.

13. Alkhouli M, Nishimura RA, Alqahtani F, Simard T, Pislaru S, Schaff H V. Predictors of use and outcomes of mechanical valve replacement in the United States (2008-2017). J Am Heart Assoc 2021;10(9).

14. Butter C, Kühnel R-U, Hölschermann F. First successful transcatheter valve-in-valve implantation into a failed mechanical prosthetic aortic valve facilitated by fracturing of the leaflets: A case report. Eur Hear J - Case Reports [Internet]. 2021 Jul 1;5(7):ytab130. Available from: https://doi. org/10.1093/ehjcr/ytab130.

15. Alperi A, Garcia S, Rodés-Cabau J. Transcatheter valve-in-valve implantation in degenerated surgical aortic and mitral bioprosthesis: Current state and future perspectives, Progress in Cardiovascular Diseases (2021), https://doi.org/ 10.1016/j. pcad.2021.10.001.

16. Pibarot P, Dumesnil JG. Prosthetic heart valves: Selection of the optimal prosthesis and long-term management. Circulation. 2009;119(7):1034-48.

17. Trasoutchi F, Montera MW, Ramos AIO, Sampaio RO, Rosa VEE, Accorsi TAD, Santi A et al. Atualização das Diretrizes Brasileiras de Valvopatias - 2020. Arq Bras Cardiol 2020;115(4):720-75.

18. Afilalo J, Lauck S, Kim DH, Lefèvre T, Piazza N, Lachapelle K, et al. Frailty in older adults undergoing aortic valve replacement: The FRAILTY-AVR Study. J Am Coll Cardiol 2017;70(6):689-700.

19. Blanke P, Weir-McCall JR, Achenbach S, Delgado V, Hausleiter J, Jilaihawi $H$ et al. Computed tomography imaging in the context of transcatheter aortic valve implantation (TAVI)/Transcatheter aortic valve replacement (TAVR): An expert consensus document of the Society of Cardiovascular Computed Tomography. JACC Cardiovasc Imaging 2019;12(1):1-24.

20. Tuzcu EM, Kapadia SR, Vemulapalli S, Carroll JD, Holmes DR, Mack MJ, et al. Transcatheter aortic valve replacement of failed surgically implanted bioprostheses: The STS/ACC Registry. J Am Coll Cardiol 2018;72(4):370-82.

21. Bleiziffer S, Simonato M, Webb JG, Rodés-Cabau J, Pibarot P, Kornowski R et al. Long-term outcomes after transcatheter aortic valve implantation in failed bioprosthetic valves. Eur Heart J 2020;41(29):273142. 
22. Dauerman HL, Deeb GM, O'Hair DP, Waksman R, Yakubov SJ, Kleiman NS et al. Durability and clinical outcomes of transcatheter aortic valve replacement for failed surgical bioprostheses. Circ Cardiovasc Interv 2019;12(10):1-12.

23. Ribeiro HB, Rodés-Cabau J, Blanke P, Leipsic J, Kwan Park J, Bapat V et al. Incidence, predictors, and clinical outcomes of coronary obstruction following transcatheter aortic valve replacement for degenerative bioprosthetic surgical valves: Insights from the VIVID registry. Eur Heart J 2018;39(8):687-95.

24. Khan JM, Greenbaum AB, Babaliaros VC, et al. The BASILICA trial: Prospective multicenter investigation of intentional leaflet laceration to prevent TAVR coronary obstruction. JACC Cardiovasc Interv 2019;12(13):12401252. doi:10.1016/j.jcin.2019.03.035.
25. Dayan V, Vignolo G, Soca G, Paganini JJ, Brusich D, Pibarot P. Predictors and outcomes of prosthesispatient mismatch after aortic valve replacement. JACC Cardiovasc Imaging 2016;9(8):924-933. doi:10.1016/j. jcmg.2015.10.026.

26. Deharo P, Bisson A, Herbert J, Lacour T, Etienne C Saint, Porto A, et al. Transcatheter valve-in-valve aortic valve replacement as an alternative to surgical re-replacement. J Am Coll Cardiol 2020;76(5):48999.

27. Sá MPBO, Van den Eynde J, Simonato M, Cavalcanti LRP, Doulamis IP, Weixler V et al. Valvein-valve transcatheter aortic valve replacement versus redo surgical aortic valve replacement: An updated meta-analysis. JACC Cardiovasc Interv 2021;14(2):211-20. 